OPEN ACCESS

Edited by:

Lucia Lopalco,

San Raffaele Hospital (IRCCS), Italy

Reviewed by:

Geng Li,

Jinan University, China

Zhenlong Liu,

McGill University, Canada

*Correspondence:

Wei-Ting Liao

wtliao@kmu.edu.tw

Yen-Hsu Chen

infchen@gmail.com

${ }^{\dagger}$ These authors have contributed equally to this work

Specialty section:

This article was submitted to

Viral Immunology,

a section of the journal

Frontiers in Immunology

Received: 27 August 2020 Accepted: 11 January 2021

Published: 15 February 2021

Citation:

Lin C-Y, Huang C-H, Wang W-H,

Tenhunen J, Hung L-C, Lin C-C, Chen Y-C, Chen Y-H and Liao W-T (2021) Mono-(2-ethylhexyl) phthalate Promotes Dengue Virus Infection by

Decreasing IL-23-Mediated Antiviral Responses.

Front. Immunol. 12:599345. doi: 10.3389/fimmu.2021.599345

\section{Mono-(2-ethylhexyl) phthalate Promotes Dengue Virus Infection by Decreasing IL-23-Mediated Antiviral Responses}

\author{
Chun-Yu Lin ${ }^{1,2,3}$, Chung-Hao Huang ${ }^{1,2}$, Wen-Hung Wang ${ }^{1,2}$, Jyrki Tenhunen ${ }^{3}$, \\ Ling-Chien Hung ${ }^{1,2}$, Chi-Chou Lin ${ }^{4}$, Yu-Cheng Chen ${ }^{1,2}$, Yen-Hsu Chen ${ }^{2,5,6 *}$ \\ and Wei-Ting Liao ${ }^{4,7,8 * T}$
}

\begin{abstract}
1 Division of Infectious Diseases, Department of Internal Medicine, Kaohsiung Medical University Hospital, Kaohsiung Medical University, Kaohsiung, Taiwan, 2 School of Medicine, Graduate Institute of Medicine, College of Medicine, Center for Tropical Medicine and Infectious Diseases Research, Kaohsiung Medical University, Kaohsiung, Taiwan, ${ }^{3}$ Department of Surgical Sciences, Department of Medical Biochemistry and Microbiology, Uppsala University, Uppsala, Sweden, ${ }^{4}$ Department of Biotechnology, College of Biomedical Science, Kaohsiung Medical University, Kaohsiung, Taiwan, ${ }^{5}$ Department of Internal Medicine, Kaohsiung Municipal Ta-Tung Hospital, Kaohsiung, Taiwan, ${ }^{6}$ Department of Biological Science and Technology, College of Biological Science and Technology, National Chiao Tung University, HsinChu, Taiwan, ${ }^{7}$ Department of Medical Research, Kaohsiung Medical University Hospital, Kaohsiung Medical University, Kaohsiung, Taiwan, ${ }^{8}$ Research Center for Environmental Medicine, Kaohsiung Medical University, Kaohsiung, Taiwan
\end{abstract}

Exposure to environmental hormones such as di(2-ethylhexyl) phthalate (DEHP) has become a critical human health issue globally. This study aimed to investigate the correlations between DEHP/mono-(2-ethylhexyl) phthalate (MEHP) levels and macrophage-associated immune responses and clinical manifestations in dengue virus (DV)-infected patients. Among 89 DV-infected patients, those with DV infection-related gastrointestinal (Gl) bleeding ( $n=13,15 \%$ of patients) had significantly higher DEHP exposure than those without Gl bleeding ( $\mathrm{n}=76,85 \%$ of patients), which were $114.2 \mathrm{ng}$ / $\mathrm{ml}$ versus $52.5 \mathrm{ng} / \mathrm{ml} \Sigma \mathrm{DEHP}$ in urine; $\mathrm{p}=0.023$ ). In an in vitro study using cultured human monocyte-derived macrophages (MDMs) to investigate the effects of MEHP, treatment increased IL-1 $\beta$ and TNF- $\alpha$ release but decreased IL-23 release, with negative correlations observed between urine $\Sigma$ DEHP and serum IL-23 levels in patients. MEHPtreated MDMs had lower antiviral Th17 response induction activity in mixed T-cell response tests. The in vitro data showed that MEHP increased DV viral load and decreased IL-23 release dose-dependently, and adding IL-23 to MEHP-exposed MDMs significantly reduced the DV viral load. MEHP also suppressed IL-23 expression via the peroxisome proliferator-activated receptor-gamma (PPAR- $\gamma$ ) pathway. Further, the PPAR- $\gamma$ antagonist GW9662 significantly reversed MEHP-induced IL-23 suppression and reduced the DV viral load. These study findings help to explain the associations between high MEHP levels and the high global burden of dengue disease.

Keywords: phthalate, dengue virus, macrophage, IL-23, biological sciences 


\section{INTRODUCTION}

Dengue fever is caused by dengue virus (DV) infection mediated by a mosquito (Aedes aegypti or Aedes albopictus) bite and occurs predominantly in tropical and subtropical areas. The World Health Organization (WHO) reports that the prevalence of dengue fever has increased continuously for nearly 30 years in more than 100 countries (1). The clinical manifestations of DV infection might include asymptomatic presentation, mild fever, typical dengue rash, severe dengue hemorrhagic fever (DHF), and dengue shock syndrome (DSS). The WHO estimates that 2.5 billion people are at risk of DV infection, and 390 million people are infected (2). Among those infected, 96 million show clinical manifestations, and 0.5 million are identified as having DHF (3).

Along with global climate change, DV infection has become a major threatening to public health all around the world (4). In 2014 , a severe outbreak (> 15,000 cases) of dengue fever occurred in southern Taiwan after an illegal di(2-ethylhexyl) phthalate (DEHP) food additive incident that affected a large percentage of the national population (5). During the outbreak, more than 120 DHF cases and nearly 20 deaths were reported at Kaohsiung Medical University Hospital. Since the safety of a dengue vaccine for humans is currently controversial (6), it is imperative to clarify the risk factors associated with severe DV infection. Phthalate exposure has been reported to alter macrophage functions (7) and, therefore, probably contributes to the development of an increasing number of DV-related infectious complications (8).

DEHP is a compound used in large quantities in polyvinyl chloride (PVC) processing industries to increase the flexibility of PVC plastics. Therefore, DEHP exposure is a global environmental problem that occurs commonly via the daily usage of plastic food/drink containers and plastics used in medical equipment. The estimated average human DEHP intake in the general population is $0.71-4.6 \mu \mathrm{g} / \mathrm{kg} / \mathrm{day}$ (9). However, among hemodialysis patients, exposure might average $75.2 \mathrm{mg}$ of DEHP during a single dialysis session (10). After exposure, DEHP rapidly converts to the phthalate metabolite mono-(2-ethylhexyl) phthalate (MEHP) in the human body via the phase I xenobiotic metabolic process in the liver (11-13). MEHP alters macrophage functions, including increased TNF- $\alpha$ and prostaglandin secretion $(7,14)$.

Primary human splenic macrophages are the major target cells for DV $(15,16)$. Surface receptors on macrophages are required for DV infection, including ICAM3-grabbing nonintegrin receptors, DC-SIGN, L-SIGN, high-affinity laminin receptor, mannose receptor, and glucose-regulated protein 78 (GRP78). After infection is established, the macrophages release many cytokines, including IFN- $\gamma$, TNF- $\alpha$, IL-8, IL-6, IL-1 $\beta$, and IL-23, which leads to different pathologic outcomes (17-19). For example, TNF- $\alpha$ and IL-8 increase the permeability of the vessel endothelial cells, resulting in vascular leakage and shock (DHF/ DSS) (20). In contrast, IL-23 released from DV-infected macrophages initiates antiviral responses by activating Th17associated responses (21).

Therefore, the present study aimed to determine the correlations, as well as underlying mechanisms, between phthalate compound exposure and DV infection using IRBproven (KMUH-IRB-20140303) samples from the 2014 DV outbreak. We hypothesized that since MEHP might alter macrophage cytokine expression and responses, it could also change the outcomes of DV infection. Clinical investigations were conducted on DV-infected patients and on MEHP-exposed human monocyte-derived macrophages (MDMs) to test this hypothesis and investigate the biological effects of MEHP exposure on macrophages. Since plastic items are used globally, and phthalate contamination is almost unavoidable, understanding the interactions between MEHP and DVinfection might provide new evidence-based insights into this modern health issue.

\section{MATERIALS AND METHODS}

\section{Ethics Statement}

The human study protocol of the present study was approved by the Institutional Review Board (IRB) of Kaohsiung Medical University Hospital (IRB Number: KMUH-IRB-20140303). Signed informed consent was obtained from all subjects.

\section{Patient Enrollment}

Patients aged $\geq 20$ years were enrolled prospectively, and urine samples (to measure DEHP exposure; see the following section) were provided on the first day of hospital consultation. The time of symptom onset for each patient was recorded immediately after enrollment to minimize recall bias. Patients who visited the hospital more than 6 days after symptom onset were excluded. All patients were diagnosed with dengue fever, and all methods used for the management of the enrolled patients were in accordance with the 2009 WHO guidelines (1). WBC counts and platelet counts from peripheral blood samples were measured at least every other day until patient recovery. Patients' demographic and clinical data, including preexisting concomitant diseases, clinical manifestations, and laboratory data, were collected using standardized data collection forms.

\section{Diagnosis of DV Infection}

All laboratory diagnostic tests for the identification of DV infection were performed at laboratories accredited by the Centers for Disease Control, Department of Health, Taiwan. The diagnosis of dengue fever was confirmed by at least one positive result of real-time polymerase chain reaction (RT-PCR) or a positive test for dengue nonstructural protein 1 (NS1) Ag STRIP (Bio-Rad Laboratories, Marnes-la-Coquette, France) (4). Dengue viral RNA from patient serum was extracted using a Viral RNA mini kit (Qiagen, Cat. No. 52906, Chatsworth, CA, USA) according to the manufacturer's instructions. All dengue viral RNAs were treated with RQ1 RNase-free DNase I before being used as templates for cDNA synthesis. For reverse transcription, the PrimeScript reagent kit (TaKaRa, Cat. No. RR037A, Kyoto, Japan) was used according to the manufacturer's instructions. The resulting cDNA was evaluated for dengue viral serotypes by RT-PCR (Applied Biosystems, 
Foster 7500, CA, USA). The primers and probes used are listed in Supplementary Table S1.

\section{Cytokine Profiles of Patients With Dengue}

The serum levels of several cytokines were measured in some patients using commercial kits (cat no. 560484 and 552990, BD Biosciences, San Jose, CA, USA), following the manufacturer's instructions, including IL-1 $\beta$, IL-18, IL-23, IL-17, INF- $\gamma$, TNF- $\alpha$, IL-10, IL-6, IL-4, IL-2, IP-10, MCP-1, MIG, RANTES, and IL-8.

\section{Measurement of DEHP Exposure in Urine Samples}

Urine samples from all patients were collected in polypropylene containers after enrollment and then transferred to an acetonitrileprewashed amber glass bottle. The samples were stored at $-20^{\circ} \mathrm{C}$ until analysis. Phthalate metabolites were analyzed using columnswitching high-performance liquid chromatography tandem mass spectrometry (LC-MS/MS) (22). In brief, this method starts with the digestion of proteins in the mixture; the resulting peptides are separated by liquid chromatography (LC) and identified by tandem mass spectrometry (MS). Equivalent DEHP exposure was measured as previously described (23). The summary DEHP ( $\mathrm{DEEHP}$ ) metabolite measurement was calculated based on the molar sum of MEHP, MEOHP, MEHHP, and MECPP, according to the following formula:

( weight concentration of MEHP/278.3) + (MEOHP/292.3)

$$
+(\mathrm{MEHHP} / 294.3)+(\mathrm{MECPP} / 308.3)] \times 390.6(23) .
$$

\section{Human Monocyte-Derived Macrophages}

The in vitro part of this study was approved by the Institutional Review Board of Kaohsiung Medical University (KMUH-IRB20140303). Normal human primary CD14+ monocytes were isolated from peripheral blood mononuclear cells by magnetic bead sorting with an anti-CD14 monoclonal antibody (MACS, Miltenyi Biotec, Germany) according to the manufacturer's instructions (24). The isolated CD14+ cells were first incubated in a culture medium with $100 \mathrm{ng} / \mathrm{ml}$ of recombinant human GMCSF for 3 days, followed by stimulation with $10 \mathrm{ng} / \mathrm{ml}$ LPS and $50 \mathrm{ng} / \mathrm{ml}$ IFN- $\gamma$ for 3 additional days to obtain MDMs. MDMs $\left(10^{6} / \mathrm{ml}\right)$ were cultured in RPMI 1640 medium with $10 \%$ FBS at $37^{\circ} \mathrm{C}$ in a humidified incubator with $5 \% \mathrm{CO}_{2}$ for NIR treatments.

\section{Enzyme-Linked Immunosorbent Assay}

After MEHP treatment in vitro, cell-free supernatants from cultured MDMs were obtained to test the concentrations of cytokines by ELISA. Human IL-1 $\beta$, IL-6, IL-8, TNF- $\alpha$, IL-10, IL-12, IL-23, IFN- $\gamma$, IL-13, and IL-17 ELISA kits (R\&D Systems, Minneapolis, MN, USA) were used according to the manufacturer's instructions. Chemokine or cytokine concentrations were calculated based on linear regression standard curves, in which $\mathrm{r}^{2}$ was higher than 0.99 .

\section{Infection and Quantification of the DV RNA}

MDMs at a density of $1 \times 10^{5}$ cells $/ \mathrm{ml}$ were infected with $\mathrm{DV}$ at a multiplicity of infection of 0.1 for 2 hours. After the infection, the supernatant was removed, and the cells were incubated with various concentrations of MEHP for an additional 2 days.

For the quantification of DV RNA, total cellular RNA from treated MDMs was extracted using the Total RNA Miniprep Purification Kit (GMbiolab, Taiwan) according to the manufacturer's instructions. The expression level of DV NS5 mRNA was determined by quantitative real-time RT-PCR (qRTPCR) with specific primers corresponding to the DV NS5 gene as follows: forward primer, 5-AAG GTG AGA AGC AAT GCA GC-3, and reverse primer, 5-CCA CTC AGG GAG TTC TCT CT-3. The copy number of NS5 in each in vitro sample was normalized to the endogenous reference gene glyceraldehydes-3phosphate dehydrogenase (GAPDH), with the following primers: forward primer, 5-GTC TTC ACC ATG GAG AA-3, and reverse primer, 5-ATG GCA TGG ACT GTG GTC AT-3. The CT value of each sample was determined using the ABI Step One Real-Time PCR System (ABI Warrington, UK).

\section{PPAR $\gamma$ Luciferase Activity Assay}

A commercially obtained peroxisome proliferator-activated receptor-gamma (PPAR $\gamma$ ) promoter firefly luciferase vector was used to detect PPAR $\gamma$ promoter activity (with Renilla luciferase as an internal control). Cells grown in 24-well plates were transfected with $1 \mu \mathrm{g}$ LipofectAMINE 3000 reagent, $4 \mu \mathrm{l}$ LipofectAMINE-Plus reagent (Invitrogen, Carlsbad, CA), $10 \mathrm{ng}$ Renilla control vector, and $80 \mathrm{ng}$ of PPAR $\gamma$ firefly vector (Clonetech, Palo Alto, CA). The cells were incubated in the aforementioned mixture for $6 \mathrm{~h}$ at $37^{\circ} \mathrm{C}$. After incubation, the transfection mixture was exchanged with a culture medium with or without MEHP treatment. Luciferase activity was measured using the Dual-Luciferase Reporter Assay system (Promega, Madison, WI) according to the manufacturer's protocol.

\section{Statistical Analysis}

Categorical data are presented as numbers; the chi-square test or Fisher's exact test was performed for categorical data. Univariate analyses were performed to determine which factors or manifestations were associated with high or low phthalate exposure. Continuous variables were analyzed by Student's ttests unless otherwise described. Statistical analyses were performed using IBM SPSS statistical software version 22 for Windows (IBM Corp., Armonk, New York, USA). A two-tailed p-value $<0.05$ was considered statistically significant. For in vitro experimental samples, a two-tailed Kruskal-Wallis test was used to calculate significance. All error bars in the graphs indicate the standard deviation (SD).

\section{RESULTS}

\section{DV-Infected Patients With High Urine IDEHP Concentrations Are at a Higher Risk of Chills and Gastrointestinal Bleeding}

A total of $89 \mathrm{DV}$-infected patients in a medical center in Southern Taiwan were enrolled between August and December 2014. The patients' demographic and clinical characteristics are shown in 
Table 1. Further analysis of dengue patients revealed typical dengue symptoms such as chills, headache, myalgia, and gastrointestinal (GI) bleeding. Patients with chills and GI bleeding showed significantly higher levels of DEHP exposure (without chills $=55.4$ $\mathrm{ng} / \mathrm{ml} \Sigma \mathrm{DEHP}$ in urine versus chills $=114.3 \mathrm{ng} / \mathrm{ml} ; \mathrm{p}=0.035$; without GI bleeding $=52.5 \mathrm{ng} / \mathrm{ml}$ $\Sigma \mathrm{DEHP}$ in urine versus with GI bleeding $=114.2 \mathrm{ng} / \mathrm{mL} ; \mathrm{p}=0.023$; Figure 1). However, upon dividing patients into "low DEHP exposure" and "high DEHP exposure" groups according to the median value of $\Sigma \mathrm{DEHP}(35.3$ $\mathrm{ng} / \mathrm{ml}$ ), a significantly higher proportion of DV patients in the highexposure group experienced chills and GI bleeding, suggesting that DEHP exposure is correlated with more clinical complications, and at least DV infection-associated chills and GI bleeding.

\section{MEHP Decreases IL-23 Expression in Cultured Human MDMs and DV-Infected Patients}

We performed in vitro MEHP cytotoxicity screening (1 $\mathrm{nM}$ to 1 $\mathrm{mM}$ ) using commercialized Cell Proliferation XTT Assay Kit (Roche, Germany). MEHP lower than $0.1 \mathrm{mM}$ did not show cytotoxic effects, and the ID50 of MEHP in human MDM is about $0.5 \mathrm{mM}$ (Supplementary Figure S1). We selected 50, 100, and $200 \mathrm{nM}$ of MEHP as treatment dosages. Results of screening for MEHP-induced alterations in DV infection-associated macrophage cytokines/chemokines in cultured MDMs revealed that MEHP increased IL- $1 \beta$ and TNF- $\alpha$ release but decreased IL-10 and IL-23 release in human MDMs (Figure 2A). Among the 15 DV infectionassociated cytokines in patient samples (Supplementary Table S2S6), the decreased IL-23 level was validated in DV-infected patients with higher $\Sigma$ DEHP exposure (Figure 2B). In DV-infected patients, the urine $\Sigma \mathrm{DEHP}$ concentrations were negatively correlated ( $\mathrm{p}=$ 0.022) with serum IL-23. However, no correlations were found between $\Sigma$ DEHP levels and other cytokines (IL-1 $\beta$, TNF- $\alpha$, and IL10) in DV-infected patients.

\section{MEHP Decreases the Th17 Response in Cultured Human MDMs and DV-Infected Patients}

IL-23 promotes antiviral effects by activating Th17 cells. Results of mixed T-cell responses after MDM and CD4+ cell co-culture showed that IL-17 release was decreased in MEHP-treated MDM cells (Figures 3A, B). Decreased IL-17 was also observed in DV-

TABLE 1 | Demographic and clinical characteristics and laboratory data of patients with dengue virus infection.

\begin{tabular}{|c|c|c|c|c|}
\hline & \multicolumn{2}{|c|}{$\Sigma$ ¿EHP } & & \\
\hline & Low & High & \multicolumn{2}{|c|}{ Univariate } \\
\hline & $(n=45)$ & $(n=44)$ & $\begin{array}{c}\text { OR } \\
(95 \% \mathrm{Cl})\end{array}$ & $p$ value \\
\hline \multicolumn{5}{|l|}{ Demographic data } \\
\hline Sex, male & $23(51.1)$ & $29(65.9)$ & $1.9(0.7-4.8)$ & 0.230 \\
\hline Age $\geq 65$ years & $7(15.6)$ & $12(27.3)$ & $2.0(0.7-5.8)$ & 0.276 \\
\hline Median age (IQR) & $51(38-63)$ & $56(42-64)$ & & 0.173 \\
\hline Presentation time (median, day after onset) & $1(0-3)$ & $1(1-3)$ & & 0.318 \\
\hline \multicolumn{5}{|l|}{ Pre-existing medical condition } \\
\hline Peptic ulcer disease & $2(4.4)$ & $4(9.1)$ & $2.2(0.4-12.3)$ & 0.652 \\
\hline Hepatitis B virus & $10(22.2)$ & $3(6.8)$ & $0.3(0.1-1.0)$ & 0.079 \\
\hline Hepatitis C virus & $1(2.2)$ & $2(4.5)$ & $2.1(0.2-23.8)$ & 0.984 \\
\hline Hypertension & $10(22.2)$ & $12(27.3)$ & $1.3(0.5-3.4)$ & 0.759 \\
\hline Diabetes mellitus & $6(13.3)$ & $7(15.9)$ & $1.2(0.4-4.0)$ & 0.965 \\
\hline Chronic kidney disease & $1(2.2)$ & $5(11.4)$ & $5.6(0.6-49.8)$ & 0.195 \\
\hline Malignancy, solid tumors & $4(8.9)$ & $3(6.8)$ & $0.8(0.2-3.6)$ & 0.999 \\
\hline \multicolumn{5}{|l|}{ Symptoms and signs at presentation } \\
\hline Fever $>38^{\circ} \mathrm{C}$ & $45(100)$ & $44(100)$ & not available & \\
\hline Chills & $4(8.9)$ & $15(34.1)$ & $5.3(1.6-17,5)$ & $0.008^{*}$ \\
\hline Headache & $17(37.8)$ & $21(47.7)$ & $1.5(0.6-3.8)$ & 0.463 \\
\hline Retroorbital pain & $4(8.9)$ & $5(11.4)$ & $1.3(0.3-6.4)$ & 0.739 \\
\hline Bone pain & $6(13.3)$ & $14(31.8)$ & $3.0(0.9-10.2)$ & 0.067 \\
\hline Joints pain (arthralgia) & $6(13.3)$ & $5(11.4)$ & $0.8(0.2-3.4)$ & 0.968 \\
\hline Myalgia & $21(46.7)$ & $27(61.4)$ & $1.8(0.7-4.6)$ & 0.239 \\
\hline Skin rash & $18(40.0)$ & $17(38.6)$ & $0.9(0.4-2.4)$ & 0.932 \\
\hline Petechiae & $9(20.0)$ & $4(9.1)$ & $0.4(0.1-1.6)$ & 0.247 \\
\hline Abdominal pain & $10(22.2)$ & $10(22.7)$ & $1.0(0.3-3.1)$ & 0.844 \\
\hline Nausea/vomiting & $13(28.9)$ & $9(20.5)$ & $0.6(0.2-1.9)$ & 0.499 \\
\hline Gl bleeding & $2(4.4)$ & $11(25.0)$ & $7.1(1.5-34.5)$ & $0.014^{\star}$ \\
\hline One or more WSs & $17(37.8)$ & $17(38.6)$ & $1.0(0.4-2.7)$ & 0.893 \\
\hline \multicolumn{5}{|l|}{ Laboratory data throughout the illness } \\
\hline Nadir PLT $<50,000 / \mu l$ & $11(24.4)$ & $16(36.4)$ & $1.8(0.6-4.9)$ & 0.321 \\
\hline Nadir WBC $<3,000 / \mu l$ & $28(62.2)$ & $29(65.9)$ & $1.2(0.5-3.1)$ & 0.887 \\
\hline
\end{tabular}

IQR, interquartile range; Gl, gastrointestinal; WSs, warning signs; PLT, platelet; WBC, white blood cell. ${ }^{*} p<0.05$, significantly different between the low and high $\Sigma D E H P$ exposure groups.

Bold values mean that these $p$ values are $<0.05$. 

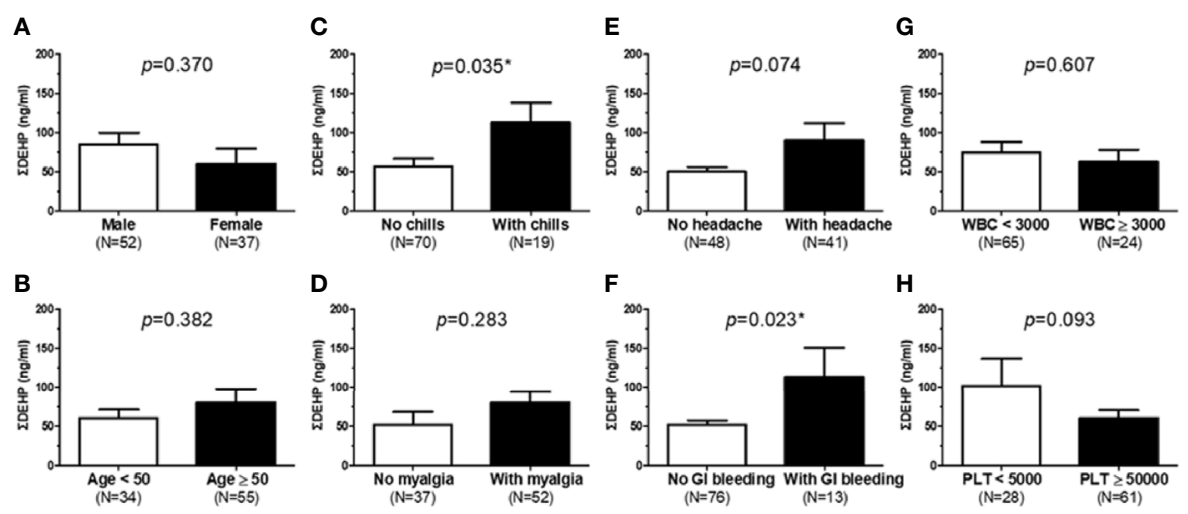

FIGURE 1 Analysis of correlations between DEHP exposure and typical symptoms in 89 dengue patients. (A, B) DEHP levels between sex and age were analyzed. (C) DEHP levels between dengue patients with or without chills. (D) DEHP levels between dengue patients with or without myalgia. (E) DEHP levels between dengue patients with or without headache. (F) DEHP levels between dengue patients with or without gastrointestinal (Gl) tract bleeding. (G) DEHP levels between white blood cell (WBC) counts (cells/ $\mu$ l) in dengue patients. (H) DEHP levels between platelet (PLT) counts (cells/ $\mu$ l) in dengue patients. Patients with chills and GI bleeding showed significantly higher levels of DEHP. ${ }^{*} \mathrm{P}<0.05$ by Student's t-test (means $\pm \mathrm{SD}$ ).

A

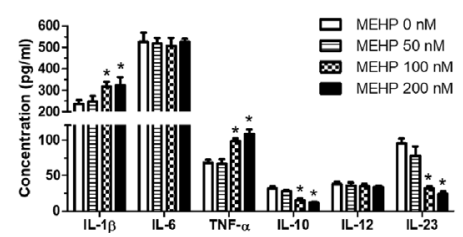

B

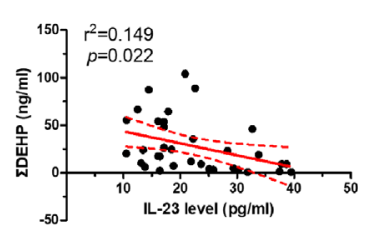

FIGURE 2 | MEHP decreases IL-23 expression in cultured human MDMs. (A) MEHP altered inflammatory cytokines in cultured human monocyte-derived macrophages (MDMs). ${ }^{*} \mathrm{P}<0.05$ MEHP-treated vs. controls by Kruskal-Wallis test; $N=8$, means $\pm \mathrm{SD}$. (B) Urine MEHP levels correlated negatively with serum IL23 levels in dengue virus (DV)-infected patients. $N=43$ by linear regression analysis.

infected patients for whom urine $\mathrm{DEHH}$ concentrations correlated negatively with serum IL-17 levels (Figure 3C), suggesting that MEHP might alter antiviral responses, at least in part, by decreasing IL-23/IL-17-mediated mechanisms.

\section{MEHP Increases DV Infection in Human MDMs}

Correlations between MEHP treatment and DV infection in MDMs revealed that MEHP treatment dose-dependently increased the viral load (Figure 4A), whereas IL-23 release from DV-infected MDMs was dose-dependently decreased (Figure 4B). Moreover, MEHP-induced DV viral load increases were significantly suppressed by applying $100 \mathrm{pg} / \mathrm{ml}$ of recombinant human IL-23 (Figure 4C), indicating that IL-23 contributes to antiviral responses in DV infection.

\section{MEHP Regulates IL-23 Expression via PPAR $\gamma$}

IL-23 production was known to be inhibited by PPAR $\gamma$ agonist (25). MEHP is known to have a U-shaped dose-response effect on PPAR $\gamma$ promoter activity $(0.1$ and $1 \mu \mathrm{M}$ MEHP showed inhibitory effects on PPAR $\gamma$, and $10 \mu \mathrm{M}$ showed activation effects) (26). To elucidate whether MEHP poses a similar effect to the PPAR $\gamma$ pathway on IL-23 regulation, we performed the following experiments. Using luciferase assays, MEHP treatment decreased PPAR $\gamma$ binding activity to the PPAR responding element (Supplementary Figure S2). In parallel, the PPAR $\gamma$ antagonist GW9662 (Sigma, St. Louis, MO) recovered the IL-23 levels and reduced the DV viral load after MEHP treatment (Figures 5A, B). These findings suggest that the PPAR $\gamma$ modulated activity of MEHP is a key regulatory mechanism of IL-23-mediated anti-DV responses.

\section{DISCUSSION}

The findings of the present study provide clues about how phthalate might affect DV-infected patients, causing them to present with obvious or less obvious symptoms or even to remain asymptomatic. Recently, DV-infected patients without symptoms (i.e., asymptomatic infection) have attracted more attention because they could present with viremia but otherwise remain asymptomatic (18). Theoretically, these asymptomatic patients will not seek medical consultation but will still be a reservoir for DV transmission. Therefore, elucidating how DVinfected patients might present with non-dominant symptoms or 


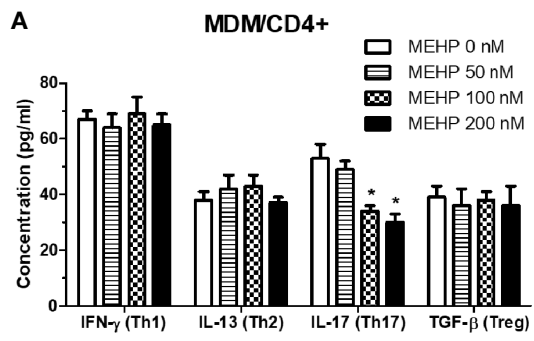

B

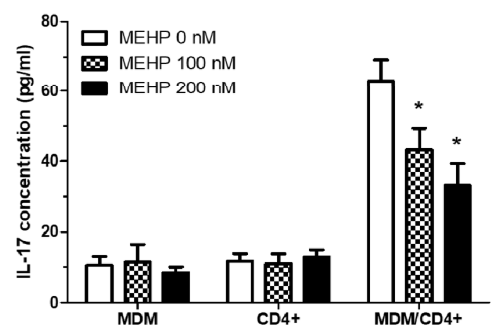

C

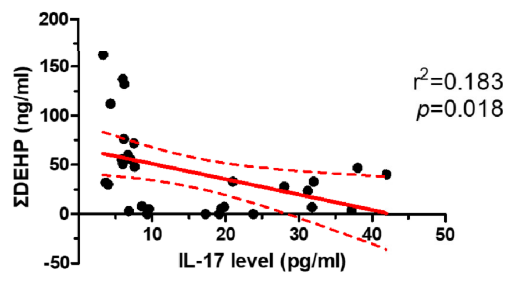

FIGURE 3 | MEHP decreases the Th17 response in cultured human MDMs. (A) Cytokines released in supernatants from co-cultured monocyte-derived macrophage (MDM)/CD4+ cells with or without MEHP treatments were tested by ELISA. ${ }^{*} \mathrm{P}<0.05 \mathrm{MEHP}$-treated vs. controls by Kruskal-Wallis test; $\mathrm{N}=8$, means $\pm \mathrm{SD}$. (B) IL-17 release in MDMs, CD4+ cells, and co-cultured MDM/CD4+ cells was tested by ELISA. *P $<0.05$ MEHP-treated vs. controls by Kruskal-Wallis test; $\mathrm{N}=3$, means $\pm \mathrm{SD}$. (C) The urine MEHP level showed a negative correlation tendency with serum IL-17 levels in dengue virus (DV)-infected patients. $\mathrm{N}=43$ by linear regression analysis.

A

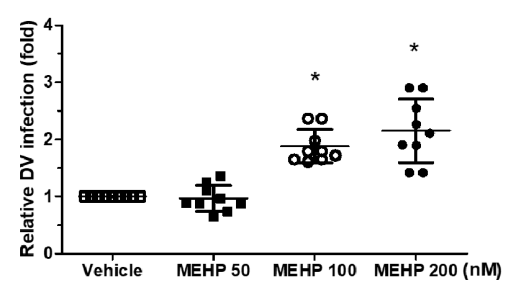

C

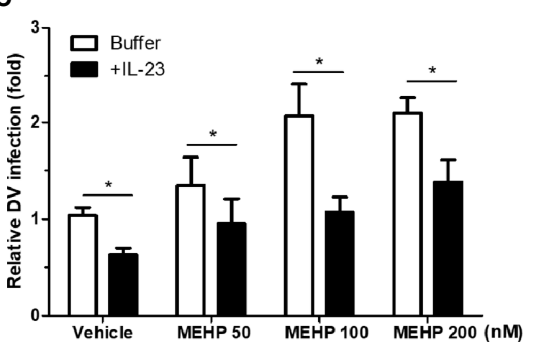

B

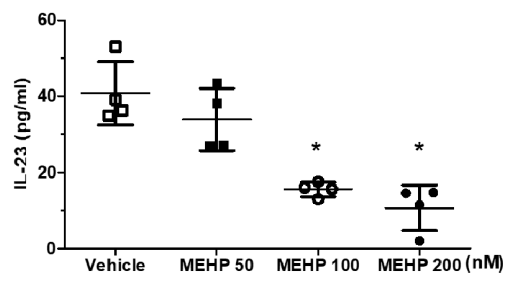

FIGURE 4 | IL-23 treatments decrease dengue virus (DV) infection in cultured human MDMs. (A) Human monocyte-derived macrophages (MDMs) were infected by DV and the relative viral counts were detected by real-time PCR. ${ }^{*} P<0.05$ by Kruskal-Wallis test; $N=9$, means $\pm S D$. (B) Human MDMs were infected by DV, and the IL-23 levels in supernatants were detected by ELISA. ${ }^{*} \mathrm{P}<0.05$ by Kruskal-Wallis test; $N=4$, means $\pm \mathrm{SD}$. (C) DV-infected human MDMs were treated with 100 $\mathrm{pg} / \mathrm{ml}$ of IL-23 and the relative viral counts were detected by real-time PCR. ${ }^{*} \mathrm{P}<0.05$ by Kruskal-Wallis test; $\mathrm{N}=4$, means $\pm S D$.

even remain asymptomatic might help to avoid such transmission. In the present study, DV-infected patients who presented with more obvious symptoms, such as chills, also had significantly higher phthalate exposure levels. Patients with higher phthalate exposure were associated with lower circulating levels of IL-23. In vitro, MEHP was found to suppress IL-23 expression via the PPAR- $\gamma$ pathway, resulting in increased viral infection. 


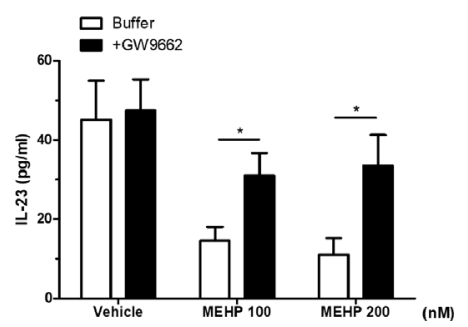

B

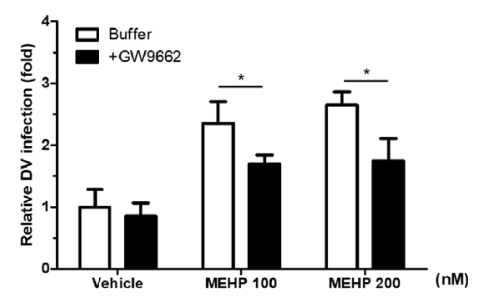

FIGURE 5 | PPAR is involved in MEHP-induced IL-23 downregulation. (A) GW9662 significantly increased IL-23 levels in MEHP-treated monocyte-derived macrophages (MDMs). (C) GW9662 significantly decreased DV viral loads in MEHP-treated MDMs. *P < 0.05 by Kruskal-Wallis test; $N=6$, means \pm SD.

The massive loss of blood is rare in vascular leakage and shock (DHF/DSS), and, when present, it is largely restricted to the gastrointestinal tract (27). In general, the presence of DV in several organs is not associated with gross or microscopic evidence of severe organ pathology, which agrees with the pathogenesis of DHF/DSS (27). Similar organ tropism has been observed in the primate model, with high concentrations of the virus isolated from the skin and gastrointestinal tract, whereas low concentrations of the virus were recovered from the spleen, thymus, and several peripheral lymph nodes (28). The authors of that study concluded that a high viral load in the blood and possibly viral tropism for endothelial cells, severe thrombocytopenia, and platelet dysfunction might result in characteristically increased capillary fragility, clinically manifested as petechiae, easy bruising, and GI mucosal bleeding. In a recent study that also included data from the 2014 Taiwan outbreak of DV-infection (4), NS1 and endothelial hyperpermeability were noted by us for their contribution to vascular leakage, which is characteristic of the disease.

In the present study, higher levels of DEHP exposure were observed in dengue patients with GI bleeding. TNF- $\alpha$ and IL- 8 are known to increase the permeability of blood vessel endothelial cells, resulting in vascular leakage. Although the present study showed that MEHP treatment increased TNF- $\alpha$ release from MDMs, no correlations were found between circulating TNF- $\alpha$ and DDEHP. Recently, other animal studies demonstrated that DV NS1 triggers localized vascular leaks in the dorsal dermis of wild-type C57BL/6 mice by altering endothelial glycocalyx components, but this was found to be independent of inflammatory cytokines (TNF- $\alpha$, IL-6, IL-8) (29, 30). Supernatants from DV-infected M1-macrophages (M1-M $\phi)$ are more potent than those from DV-infected $\mathrm{M} 2-\mathrm{M} \phi$ in terms of increasing the permeability of endothelial cells (31). Therefore, further studies are required to fully understand the interactions between MEHP and endothelial permeability.

Human $M \phi$ and dendritic cells (DCs) are the primary targets of DV infections. Unlike DCs, which undergo apoptosis during DV infection, human $\mathrm{M} \phi$ survive for at least 45 days after DV infection, suggesting that they might be regarded as a major source of proinflammatory cytokines in vivo (20,32). However, whether human inflammatory macrophage subsets display distinct reactions to DV infection has not been addressed systematically. Based on mycobacterial infection studies, M1M $\phi$ secrete high levels of IL-23 (p40/p19) but not IL-12 (p40/ p35) after mycobacterial infection, whereas a secondary signal, IFN- $\gamma$, induces IL-12p35 transcription and IL-12 production. In contrast to $\mathrm{M} 1-\mathrm{M} \phi, \mathrm{M} 2-\mathrm{M} \phi$ predominantly produce IL-10 but not IL-12 and IL-23. In addition, only M1-M $\phi$, and not M2-M $\phi$, support the Th1 response after mycobacterial infection. These results indicate that IL-23, but not IL-12, is the major type 1 cytokine produced by mycobacteria-stimulated M1-M $\phi$ (33-35).

Prominent examples show that the M protein of DV directly induces cell death in infected macrophages, and bystander cells block acute antiviral responses, contribute to local tissue damage, and attenuate the efficient progression of macrophage polarization toward an M1 phenotype. All of these processes, in turn, contribute to compromised antiviral immunity, leading to a high incidence of mortality or chronic viral persistence. The stimulation of TLRs in M1-M $\phi$ with DV can induce the activation of $\mathrm{NF}-\mathrm{KB}$ and MAPK, which promotes the transcription of a range of proinflammatory cytokines (20). NLRP3 inflammasome-activated caspase-1 further processes pro-IL-1 $\beta$ and pro-IL-18 into their mature cytokine forms, IL$1 \beta$ and IL-18. IL-1 $\beta$ can also enhance the production of IL-23 and IL-6. The released IL-1 $\beta$, IL-18, and IL-23 induce Th17/ $\gamma \delta$ T cells to produce proinflammatory cytokines, which are responsible for host immune responses against DV infection $(18,20)$. Data from the present study show that MEHP suppresses the IL-23/IL-17 axis by interacting with PPAR- $\gamma$; this is because IL-23/IL-17 plays a significant role in host immunity against DV infection. These data also show that MEHP exposure increases DV viral load. Therefore, our data suggest that MEHP functions as an inhibitory agent against host immunity during DV infection, which might lead to more severe clinical manifestations such as GI bleeding.

DEHP exposure is a contemporary global environmental issue, and, in parallel with global warming, the prevalence of DV infection continues to increase. The findings of the present study provide evidence that the DEHP metabolite MEHP alters host immunity, at least in part, during DV infection, providing new insights into the correlation between DEHP exposure and more severe outcomes of DV infection. The results of this study might provide a useful academic reference for human health in the face of these two contemporary environmental issues. 


\section{DATA AVAILABILITY STATEMENT}

The original contributions presented in the study are included in the article/Supplementary Material. Further inquiries can be directed to the corresponding authors.

\section{ETHICS STATEMENT}

The studies involving human participants were reviewed and approved by the Institutional Review Board (IRB) of Kaohsiung Medical University Hospital (IRB Number: KMUH-IRB20140303). The patients/participants provided their written informed consent to participate in this study.

\section{AUTHOR CONTRIBUTIONS}

C-YL: Conception and design, acquisition of data, analysis and interpretation of data, drafting of the manuscript, final approval of the manuscript, statistical analysis, literature research, clinical studies, experimental studies, and obtaining funding. C-HH: Data acquisition, analysis and interpretation of data, drafting of the manuscript, final approval of the manuscript, literature research, clinical studies, and experimental studies. W-HW: Data acquisition, analysis and interpretation of data, drafting of the manuscript, final approval of the manuscript, and experimental studies. JT: Analysis and interpretation of data, critical revision of the manuscript, final approval of the manuscript, administrative, technical, or material support, and supervision. L-CH: Data acquisition, drafting of the manuscript, final approval of the manuscript, administrative, and technical or material support.

\section{REFERENCES}

1. WHO. WHO Guidelines Approved by the Guidelines Review Committee. In: Dengue: Guidelines for Diagnosis, Treatment, Prevention and Control: New Edition. Geneva: World Health Organization (2009).

2. Rathore AP, Farouk FS, St John AL. Risk factors and biomarkers of severe dengue. Curr Opin Virol (2020) 43:1-8. doi: 10.1016/j.coviro.2020.06.008

3. Thomas SJ. Preventing dengue-is the possibility now a reality? $N$ Engl J Med (2015) 372(2):172-3. doi: 10.1056/NEJMe1413146

4. Lin C-Y, Kolliopoulos C, Huang C-H, Tenhunen J, Heldin C-H, Chen Y-H, et al. High levels of serum hyaluronan is an early predictor of dengue warning signs and perturbs vascular integrity. EBioMedicine (2019) 48:425-41. doi: 10.1016/j.ebiom.2019.09.014

5. Wang SF, Chang K, Loh EW, Wang WH, Tseng SP, Lu PL, et al. Consecutive large dengue outbreaks in Taiwan in 2014-2015. Emerg Microbes Infect (2016) 5(12):e123. doi: 10.1038/emi.2016.124

6. Normile D. Safety concerns derail dengue vaccination program. Science (2017) 358(6370):1514-5. doi: 10.1126/science.358.6370.1514

7. Hansen JF, Bendtzen K, Boas M, Frederiksen H, Nielsen CH, Rasmussen AK, et al. Influence of phthalates on cytokine production in monocytes and macrophages: a systematic review of experimental trials. PloS One (2015) 10 (3):e0120083. doi: 10.1371/journal.pone.0120083

8. John DV, Lin YS, Perng GC. Biomarkers of severe dengue disease - a review. J BioMed Sci (2015) 22:83. doi: 10.1186/s12929-015-0191-6

9. Chang JW, Lee CC, Pan WH, Chou WC, Huang HB, Chiang HC, et al. Estimated Daily Intake and Cumulative Risk Assessment of Phthalates in the
C-CL: Data acquisition, drafting of the manuscript, and final approval of the manuscript. Y-CC: Data acquisition, drafting of the manuscript, and final approval of the manuscript. Y-HC: Conception and design, acquisition of data, analysis and interpretation of data, critical revision of the manuscript, final approval of the manuscript, acting as a guarantor of integrity of the entire study, statistical analysis, clinical studies, obtaining funding, and supervision. W-TL: Conception and design, acquisition of data, analysis and interpretation of data, critical revision of the manuscript, final approval of the manuscript, acting as a guarantor of integrity of the entire study, statistical analysis, literature research, experimental studies, obtaining funding, and supervision. All authors contributed to the article and approved the submitted version.

\section{FUNDING}

This study was supported by grants from the Ministry of Science and Technology (MOST103-2314-B-037-068; MOST104-2314B-037-079; MOST106-2320-B-037-030-MY3; MOST107-2314B-037-087; MOST108-2314-B-037-046-), Kaohsiung Medical University (KMU-TP103A13; KMU-TP104A30; KMUTC109A01-1), and Kaohsiung Medical University Hospital (KMUH104-4R14).

\section{SUPPLEMENTARY MATERIAL}

The Supplementary Material for this article can be found online at: https://www.frontiersin.org/articles/10.3389/fimmu.2021. 599345/full\#supplementary-material

General Taiwanese after the 2011 DEHP Food Scandal. Sci Rep (2017) 7:45009. doi: 10.1038/srep45009

10. Faouzi MA, Dine T, Gressier B, Kambia K, Luyckx M, Pagniez D, et al. Exposure of hemodialysis patients to di-2-ethylhexyl phthalate. Int J Pharm (1999) 180(1):113-21. doi: 10.1016/S0378-5173(98)00411-6

11. Kim SH, On JW, Pyo H, Ko KS, Won JC, Yang J, et al. Percentage fractions of urinary di(2-ethylhexyl) phthalate metabolites: Association with obesity and insulin resistance in Korean girls. PloS One (2018) 13(11):e0208081. doi: 10.1371/journal.pone.0208081

12. Choi K, Joo H, Campbell JLJr., Clewell RA, Andersen ME, Clewell HJ. 3rd. In vitro metabolism of di(2-ethylhexyl) phthalate (DEHP) by various tissues and cytochrome P450s of human and rat. Toxicol In Vitro (2012) 26(2):315-22. doi: 10.1016/j.tiv.2011.12.002

13. Wittassek M, Angerer J. Phthalates: metabolism and exposure. Int J Androl (2008) 31(2):131-8. doi: 10.1111/j.1365-2605.2007.00837.x

14. Bolling AK, Ovrevik J, Samuelsen JT, Holme JA, Rakkestad KE, Mathisen GH, et al. Mono-2-ethylhexylphthalate (MEHP) induces TNF-alpha release and macrophage differentiation through different signalling pathways in RAW264.7 cells. Toxicol Lett (2012) 209(1):43-50. doi: 10.1016/j.toxlet.2011.11.016

15. Blackley S, Kou Z, Chen H, Quinn M, Rose RC, Schlesinger JJ, et al. Primary human splenic macrophages, but not $\mathrm{T}$ or B cells, are the principal target cells for dengue virus infection in vitro. J Virol (2007) 81(24):13325-34. doi: 10.1128/JVI.01568-07

16. Wan SW, Wu-Hsieh BA, Lin YS, Chen WY, Huang Y, Anderson R. The monocyte-macrophage-mast cell axis in dengue pathogenesis. $J$ BioMed Sci (2018) 25(1):77. doi: 10.1186/s12929-018-0482-9 
17. Fahey E, Doyle SL. IL-1 Family Cytokine Regulation of Vascular Permeability and Angiogenesis. Front Immunol (2019) 10:1426. doi: 10.3389/fimmu. 2019.01426

18. Simon-Loriere E, Duong V, Tawfik A, Ung S, Ly S, Casademont I, et al. Increased adaptive immune responses and proper feedback regulation protect against clinical dengue. Sci Transl Med (2017) 9(405):eaal5088. doi: 10.1126/scitranslmed.aal5088

19. Guzman MG, Harris E. Dengue. Lancet (2015) 385(9966):453-65. doi: 10.1016/ S0140-6736(14)60572-9

20. Wu MF, Chen ST, Hsieh SL. Distinct regulation of dengue virus-induced inflammasome activation in human macrophage subsets. J BioMed Sci (2013) 20:36. doi: 10.1186/1423-0127-20-36

21. Dungan LS, Mills KH. Caspase-1-processed IL-1 family cytokines play a vital role in driving innate IL-17. Cytokine (2011) 56(1):126-32. doi: 10.1016/ j.cyto.2011.07.007

22. Jeong JY, Lee JH, Kim EY, Kim PG, Kho YL. Determination of Phthalate Metabolites in Human Serum and Urine as Biomarkers for Phthalate Exposure Using Column-Switching LC-MS/MS. Saf Health Work. (2011) 2 (1):57-64. doi: 10.5491/SHAW.2011.2.1.57

23. Martinez RM, Hauser R, Liang L, Mansur A, Adir M, Dioni L, et al. Urinary concentrations of phenols and phthalate metabolites reflect extracellular vesicle microRNA expression in follicular fluid. Environ Int (2019) 123:208. doi: 10.1016/j.envint.2018.11.043

24. Lin CY, Wang WH, Chen SH, Chang YW, Hung LC, Chen CY, et al. Lipopolysaccharide-Induced Nitric Oxide, Prostaglandin E2, and Cytokine Production of Mouse and Human Macrophages Are Suppressed by Pheophytin-b. Int J Mol Sci (2017) 18(12):2637. doi: 10.3390/ijms18122637

25. Xu J, Drew PD. Peroxisome proliferator-activated receptor-gamma agonists suppress the production of IL-12 family cytokines by activated glia. J Immunol (2007) 178(3):1904-13. doi: 10.4049/jimmunol.178.3.1904

26. Shoaito H, Petit J, Chissey A, Auzeil N, Guibourdenche J, Gil S, et al. The Role of Peroxisome Proliferator-Activated Receptor Gamma (PPARgamma) in Mono (2-ethylhexyl) Phthalate (MEHP)-Mediated Cytotrophoblast Differentiation. Environ Health Perspect (2019) 127(2):27003. doi: 10.1289/EHP3730

27. Lee IK, Huang CH, Huang WC, Chen YC, Tsai CY, Chang K, et al. Prognostic Factors in Adult Patients with Dengue: Developing Risk Scoring Models and Emphasizing Factors Associated with Death $\leq 7$ Days after Illness Onset and $\leq 3$ Days after Presentation. J Clin Med (2018) 7(11):396. doi: 10.3390/ jcm7110396
28. Martina BE, Koraka P, Osterhaus AD. Dengue virus pathogenesis: an integrated view. Clin Microbiol Rev (2009) 22(4):564-81. doi: 10.1128/ CMR.00035-09

29. Glasner DR, Ratnasiri K, Puerta-Guardo H, Espinosa DA, Beatty PR, Harris E. Dengue virus NS1 cytokine-independent vascular leak is dependent on endothelial glycocalyx components. PloS Pathog (2017) 13(11):e1006673. doi: 10.1371/journal.ppat.1006673

30. Chen HR, Chao CH, Liu CC, Ho TS, Tsai HP, Perng GC, et al. Macrophage migration inhibitory factor is critical for dengue NS1-induced endothelial glycocalyx degradation and hyperpermeability. PloS Pathog (2018) 14(4): e1007033. doi: 10.1371/journal.ppat.1007033

31. Hsieh SL, Wu MF, Chen ST. Inflammatory macrophages in dengue virus infection pyroptosis, infectivity, and enhancement of endothelial permeability. J Immunol (2012) 188(1 Supplement):67.5.

32. Whitehead SS, Blaney JE, Durbin AP, Murphy BR. Prospects for a dengue virus vaccine. Nat Rev Microbiol (2007) 5(7):518-28. doi: 10.1038/ nrmicro1690

33. Deng J, Yu XQ, Wang PH. Inflammasome activation and Th17 responses. $\mathrm{Mol}$ Immunol (2019) 107:142-64. doi: 10.1016/j.molimm.2018.12.024

34. Sun R, Hedl M, Abraham C. IL23 induces IL23R recycling and amplifies innate receptor-induced signalling and cytokines in human macrophages, and the IBD-protective IL23R R381Q variant modulates these outcomes. Gut (2019) 69(2):264-73. doi: 10.1136/gutjnl-2018-316830

35. Wang Y, Edelmayer R, Wetter J, Salte K, Gauvin D, Leys L, et al. Monocytes/ Macrophages play a pathogenic role in IL-23 mediated psoriasis-like skin inflammation. Sci Rep (2019) 9(1):5310. doi: 10.1038/s41598-019-41655-7

Conflict of Interest: The authors declare that the research was conducted in the absence of any commercial or financial relationships that could be construed as a potential conflict of interest.

Copyright (C) 2021 Lin, Huang, Wang, Tenhunen, Hung, Lin, Chen, Chen and Liao. This is an open-access article distributed under the terms of the Creative Commons Attribution License (CC BY). The use, distribution or reproduction in other forums is permitted, provided the original author(s) and the copyright owner(s) are credited and that the original publication in this journal is cited, in accordance with accepted academic practice. No use, distribution or reproduction is permitted which does not comply with these terms. 